Supporting Information for

\title{
Interpenetrating Polymer Semiconductor Nanonetwork Channel for Ultrasensitive, Selective, and Fast Recovered Chemodetection
}

Jaehee Kim, ${ }^{\dagger}, \|$ Hyukmin Kweon, ${ }^{\dagger, \|}$ Han Wool Park, ${ }^{\dagger}, \|$ Pureunsan Go, ${ }^{\dagger}$ Haejung Hwang, ${ }^{\dagger}$ Joonseok Lee, ${ }^{\ddagger}$ Seon-Jin Choi ${ }^{\ddagger}$ and Do Hwan Kim ${ }^{*}, \dagger$

${ }^{\dagger}$ Department of Chemical Engineering, Hanyang University, Seoul 04763, Republic of Korea Division of Materials Science and Engineering, Hanyang University, Seoul 04763, Republic of Korea

*Corresponding author: dhkim76@hanyang.ac.kr

KEYWORDS: interpenetrating polymer semiconductor nanonetwork channel; chemodetection; amine receptor; ultrasensitivity and high selectivity; fast recovery 
a

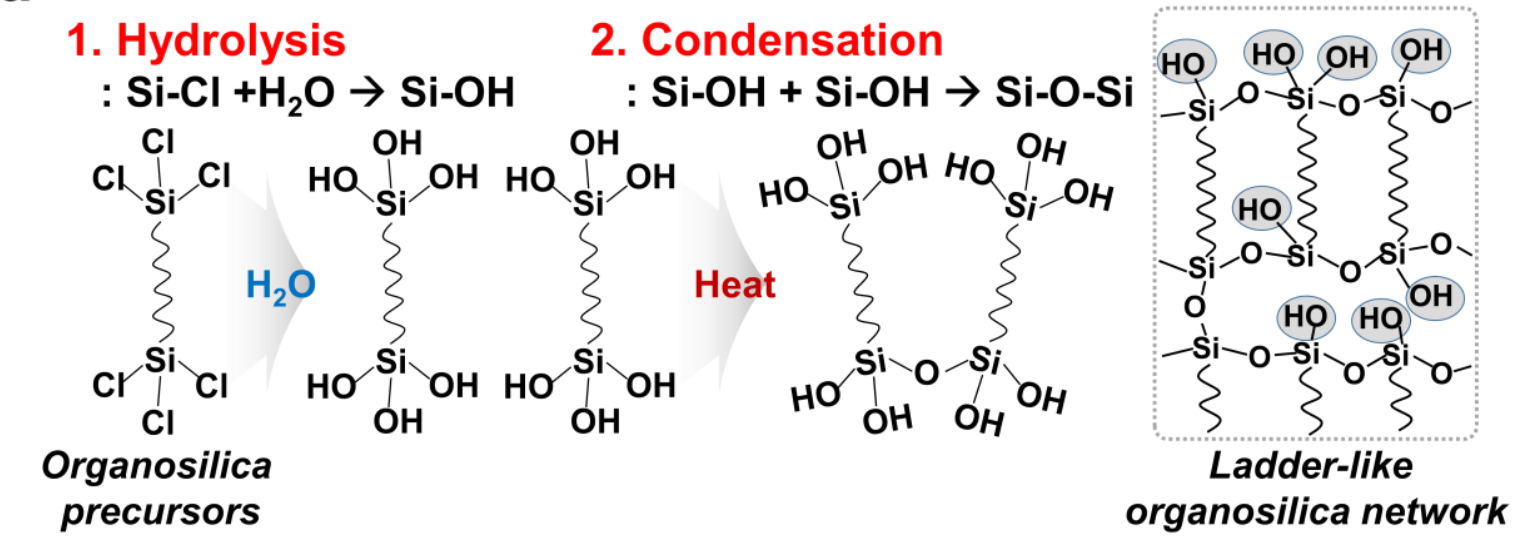

b

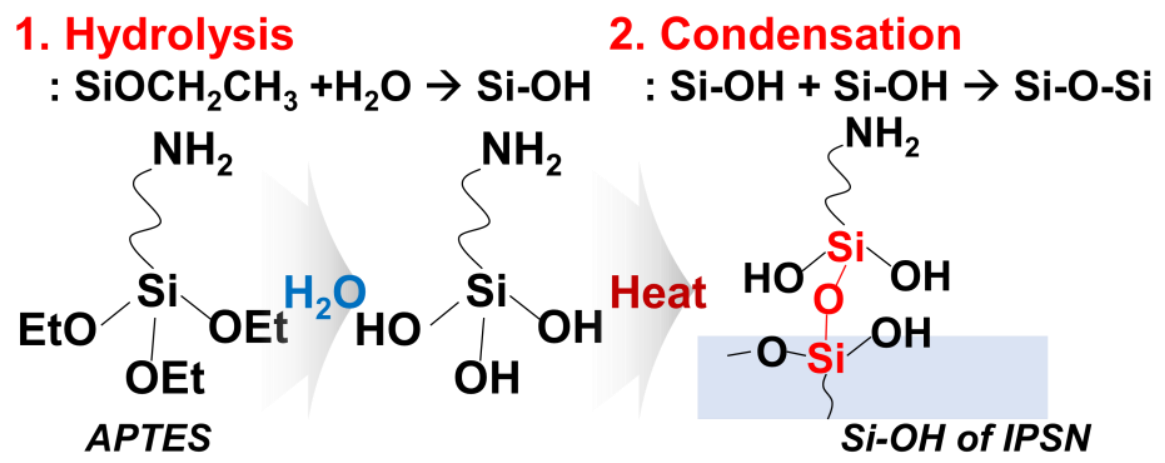

Figure S1. Schematic diagram showing sol-gel reactions. (a) Hydrolysis and condensation reactions between organosilica precursors to form a ladder-like organosilica network. (b) Hydrolysis reaction of APTES molecules and condensation reaction between $\mathrm{Si}-\mathrm{OH}$ groups in APTES and IPSN. 

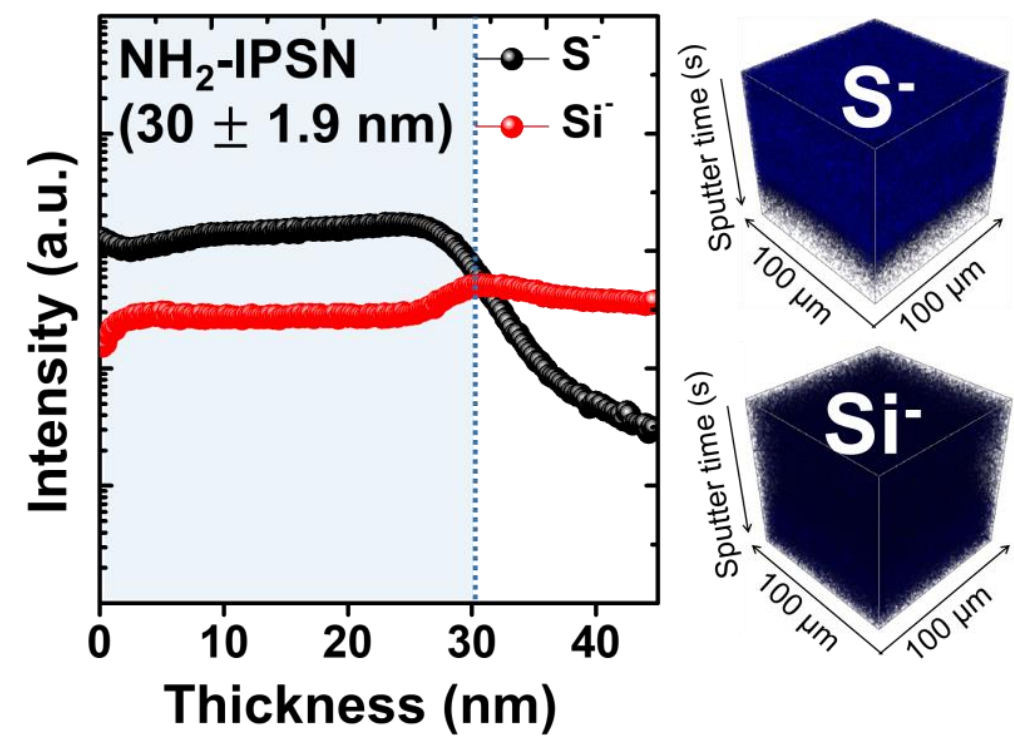

Figure S2. Negative ToF-SIMS spectra and 3D mapping $\left(\mathrm{S}^{-}\right.$and $\left.\mathrm{Si}^{-}\right)$of $\mathrm{NH}_{2}-\mathrm{IPSN}$.
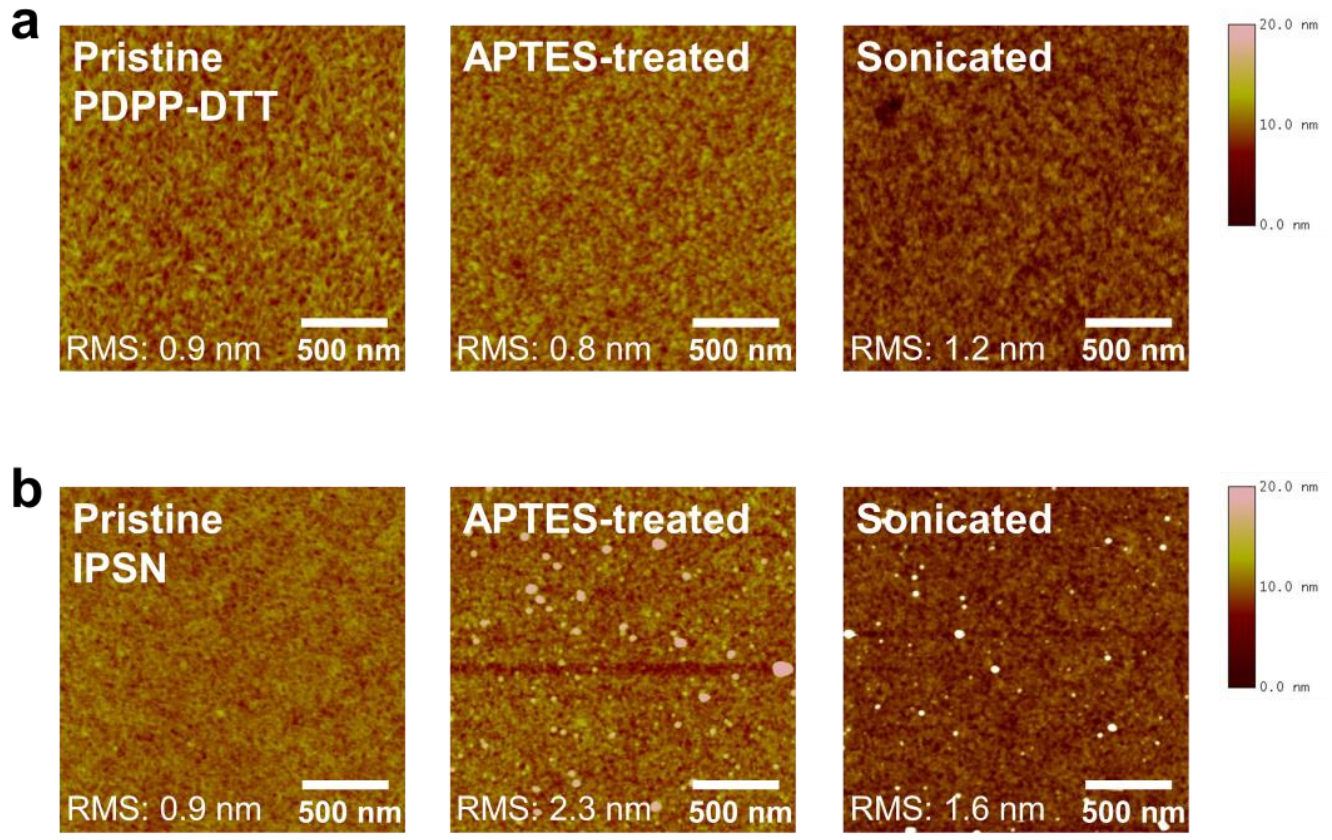

Figure S3. AFM images before and after dipping in APTES solution and sonication of (a) PDPP-DTT and (b) IPSN films. 


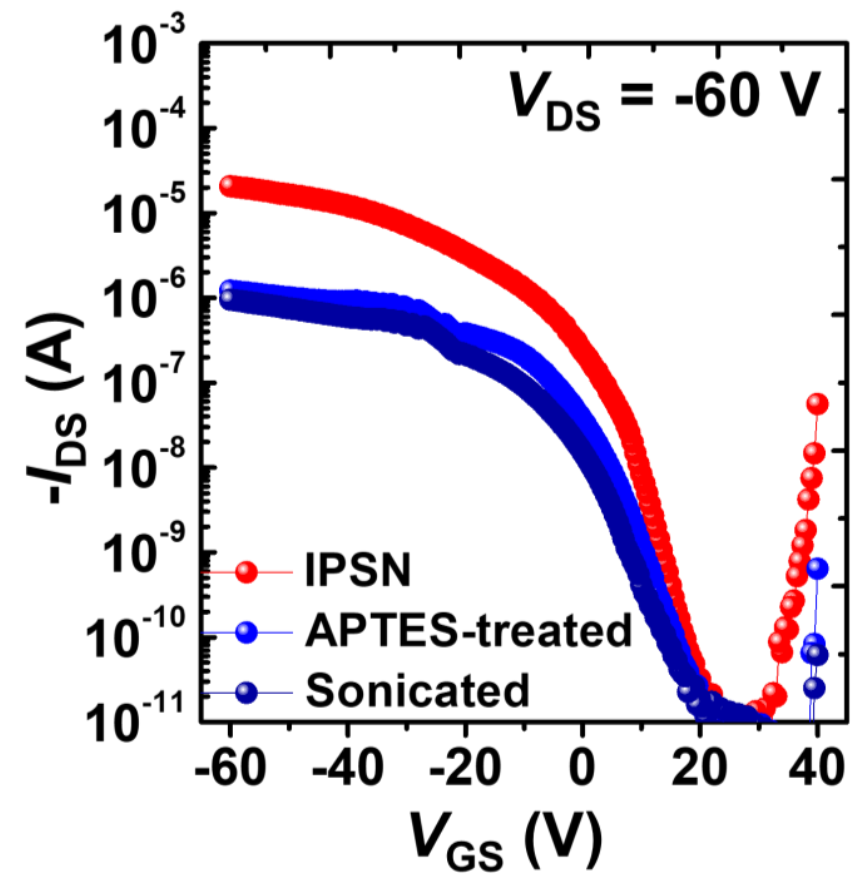

Figure S4. Transfer curves of IPSN-based FET before and after dipping in APTES solution and sonication.
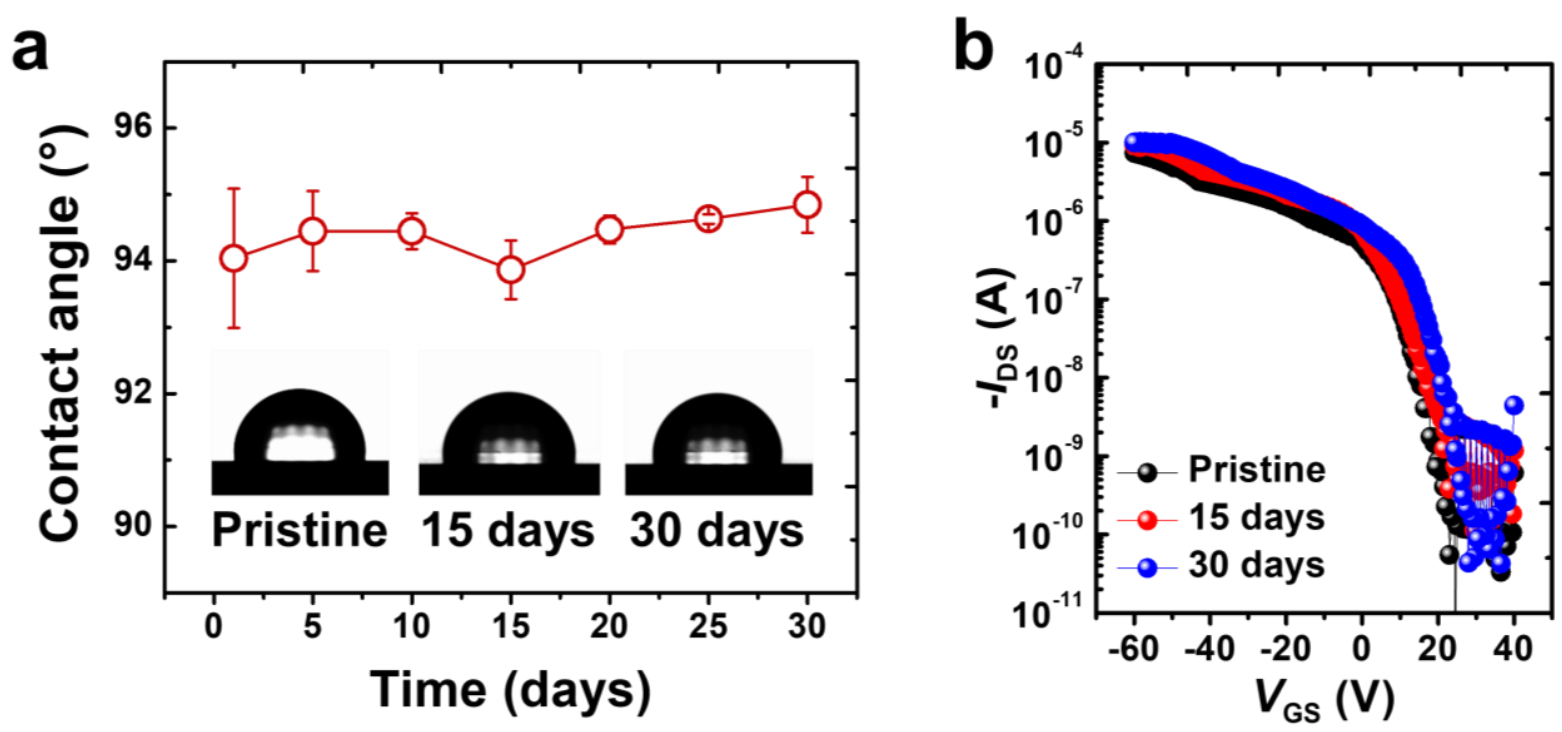

Figure S5. Evaluation of air stability of $\mathrm{NH}_{2}$-IPSN film. (a) Contact angle measurement of $\mathrm{NH}_{2}$-IPSN film storing in ambient environment for 30 days. (b) Transfer characteristics of $\mathrm{NH}_{2}$-IPSN-based FET corresponding to pristine (black), 15 days (red), and 30 days later (blue) in ambient environment, respectively. 


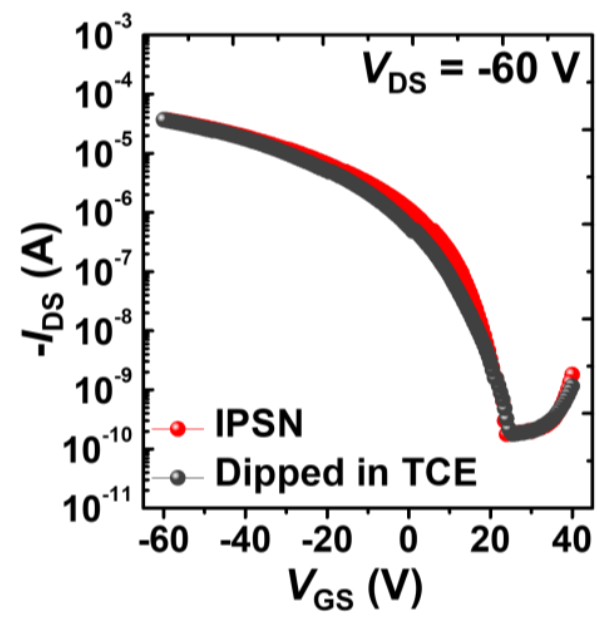

Figure S6. Transfer curves of IPSN-based FET before and after dipping in trichloroethylene (TCE).

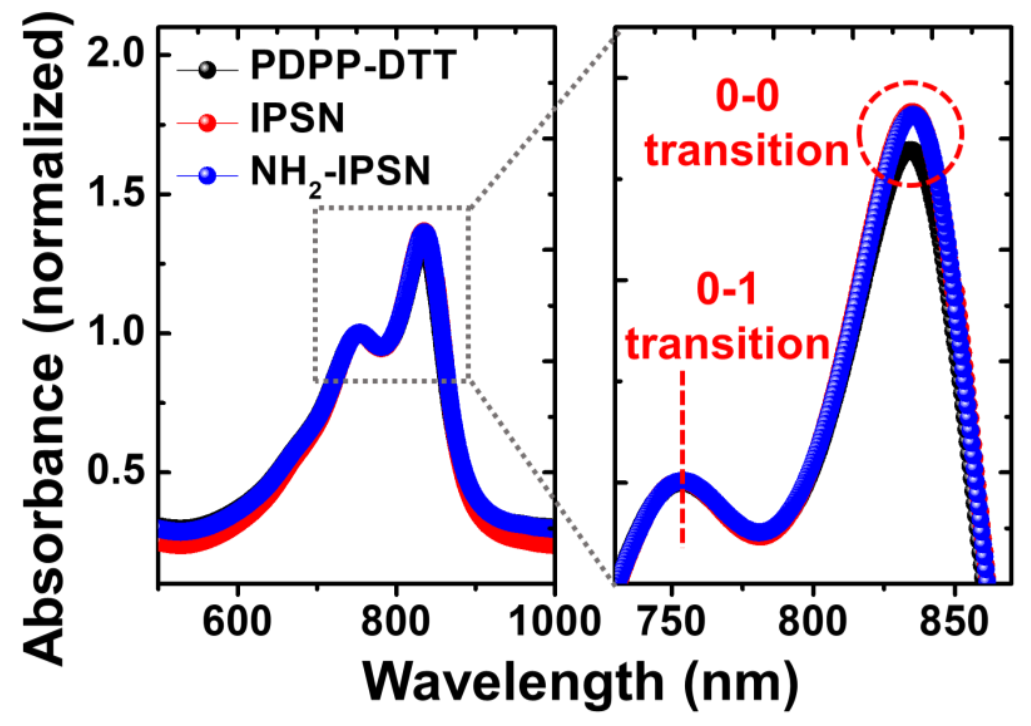

Figure S7. Normalized UV-vis-NIR spectra of PDPP-DTT, IPSN, and $\mathrm{NH}_{2}-\mathrm{IPSN}$, with magnified view of two characteristic peaks associated with the $0-1$ and $0-0$ transitions in PDPPDTT. 

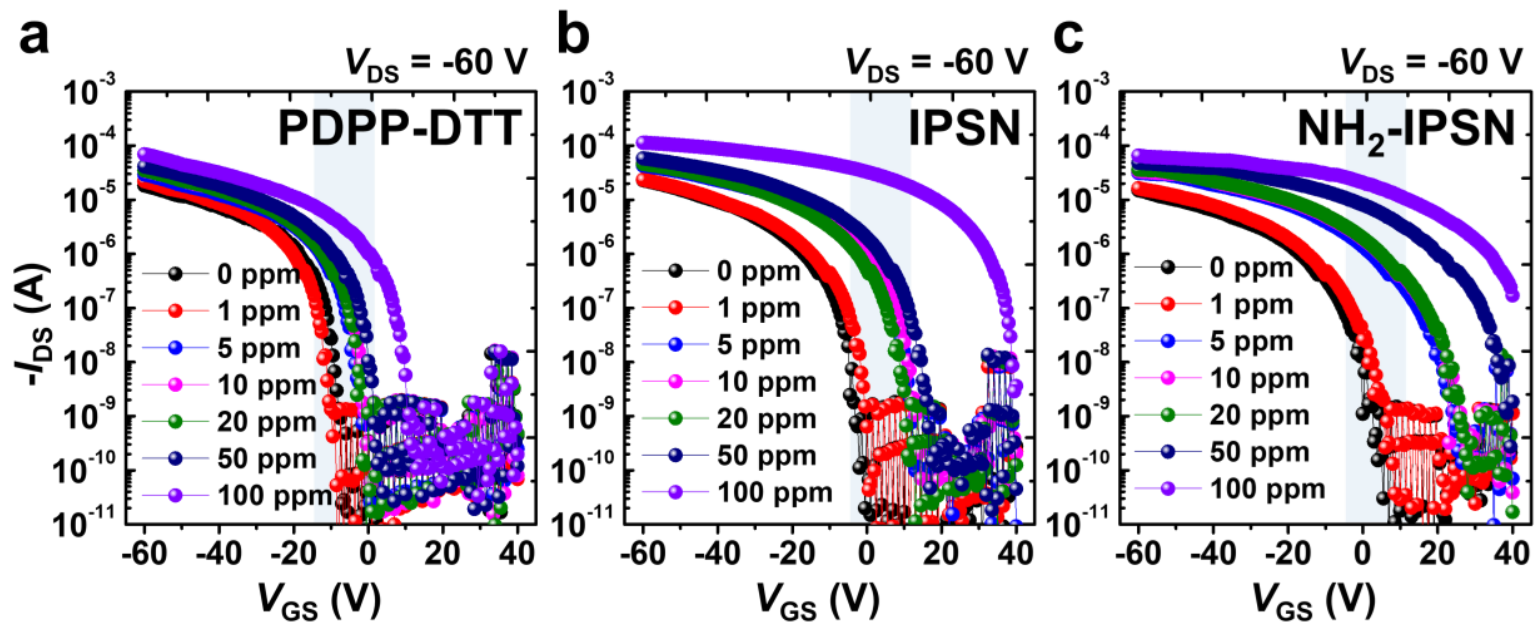

Figure S8. Transfer characteristics of (a) PDPP-DTT, (b) IPSN, and (c) $\mathrm{NH}_{2}$-IPSN based FETs at various $\mathrm{NO}_{2}$ concentrations (1-100 ppm).
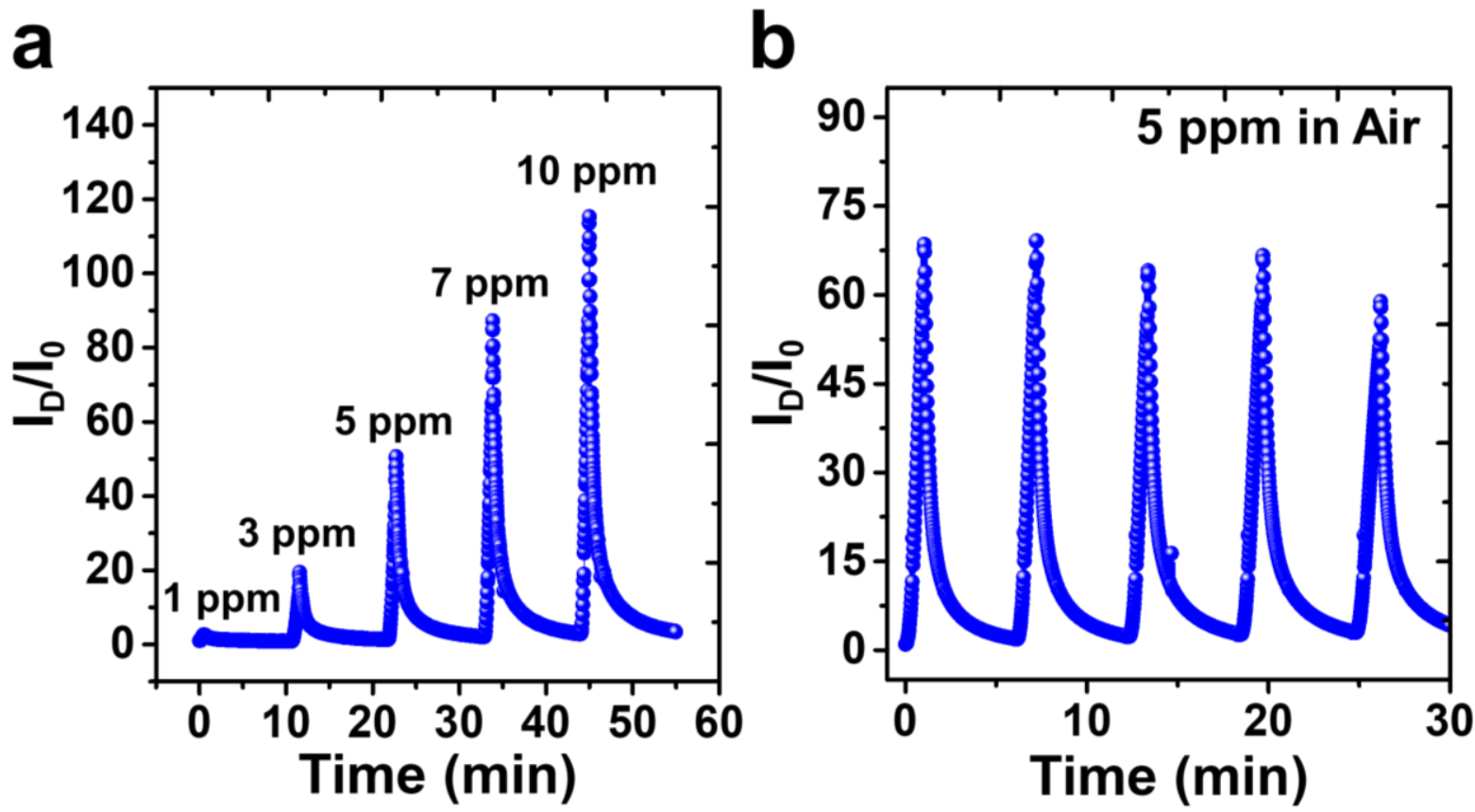

Figure S9. $\mathrm{NO}_{2}$ sensing characteristics under air condition of $\mathrm{NH}_{2}$-IPSN-based FET. (a) Dynamic sensing response of the $\mathrm{NH}_{2}$-IPSN as a function of time at $\mathrm{NO}_{2}$ concentrations ranging from 1 to $10 \mathrm{ppm}$ in air condition. (b) Multi-cyclic response characteristics of the $\mathrm{NH}_{2}$ IPSN against $5 \mathrm{ppm} \mathrm{NO}_{2}$ in air condition. 
a

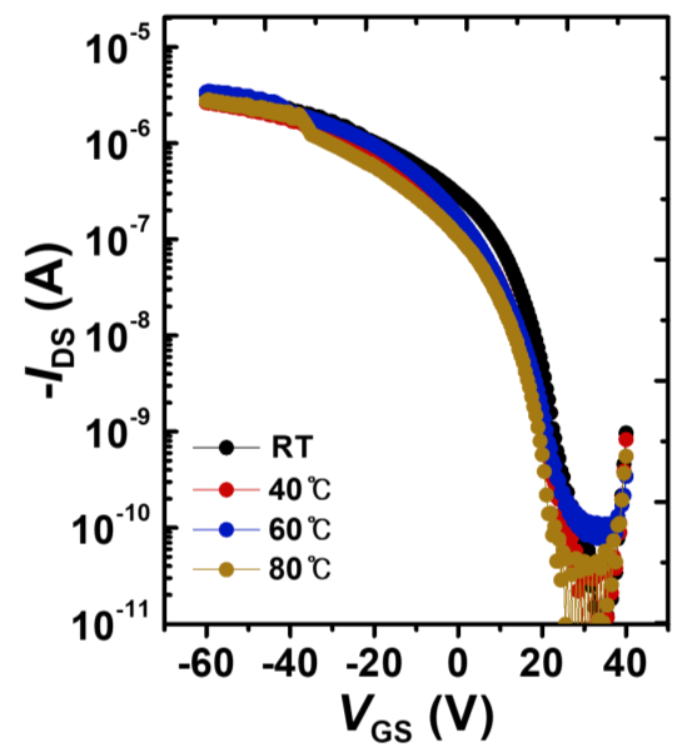

b

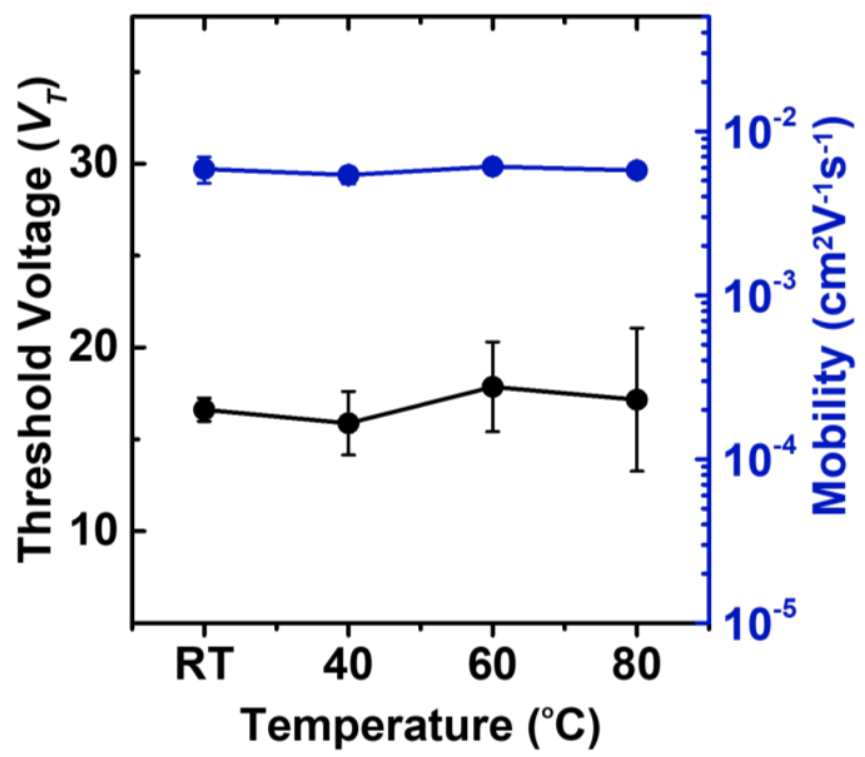

Figure S10. Temperature dependence of $\mathrm{NH}_{2}$-IPSN-based FET. (a) Transfer characteristics of $\mathrm{NH}_{2}$-IPSN-based FET depending on temperature ranging from room temperature to $80^{\circ} \mathrm{C}$. (b) Field-effect mobility and threshold voltage of $\mathrm{NH}_{2}$-IPSN as a function of the temperature.

a

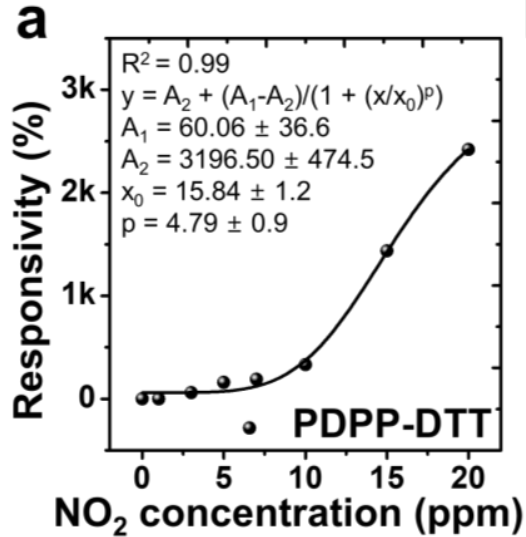

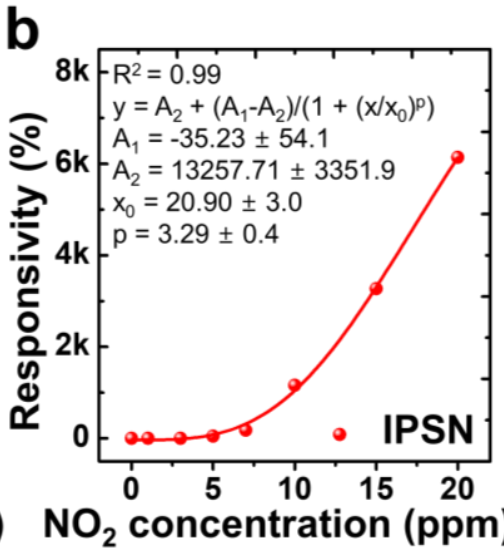

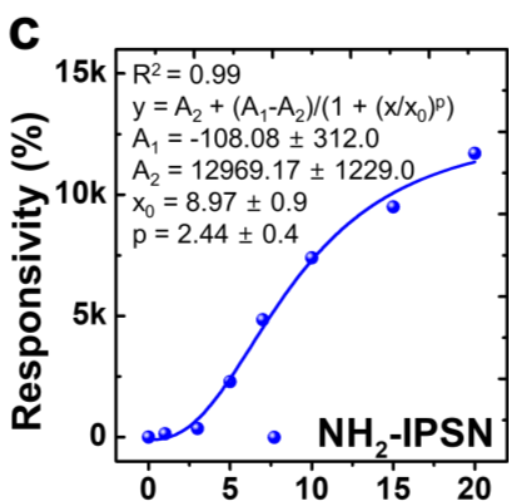

$\mathrm{NO}_{2}$ concentration (ppm)

Figure S11. Fitting curves of $\mathrm{NO}_{2}$ responsivity of (a) PDPP-DTT, (b) IPSN, and (c) $\mathrm{NH}_{2}$-IPSN based FETs by logistic regression. 